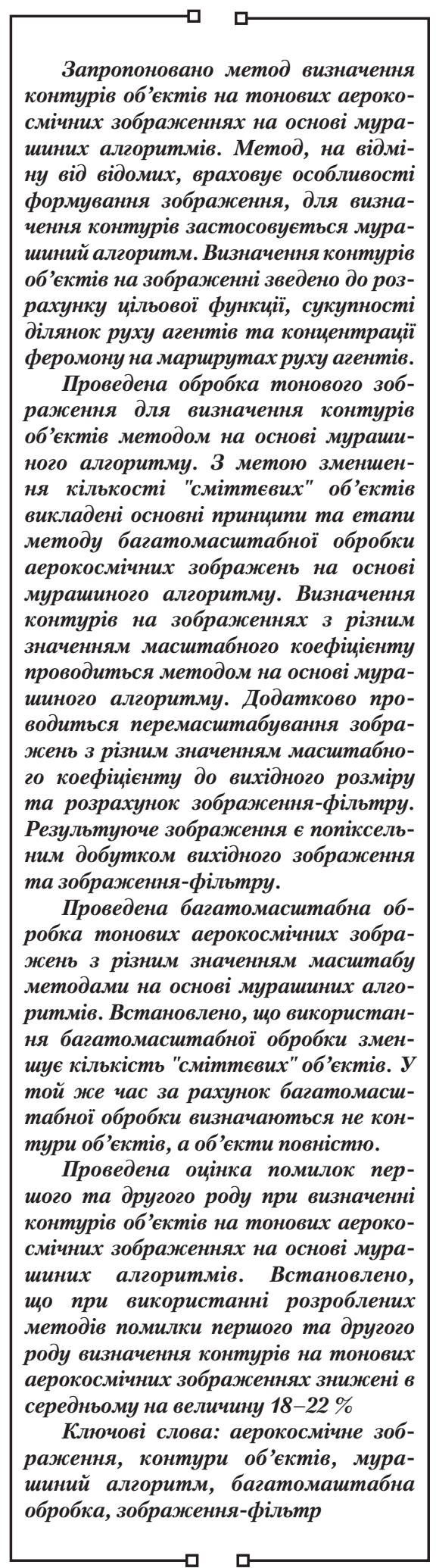

Received date 14.08.2019

Accepted date 04.09.2019

Published date 28.10.2019
UDC 004.932

DOI: $10.15587 / 1729-4061.2019 .177817$

\section{CONSTRUCTION OF METHODS} FOR DETERMINING THE CONTOURS OF OBJECTS ON TONAL AEROSPACE IMAGES BASED ON THE ANT ALGORITHMS

I. Ru b a n

Doctor of Technical Sciences, Professor, First Vice-Rector*

H. K h u d o v

Doctor of Technical Sciences, Professor, Head of Department Department of Radar Troops Tactic ** E-mail: 2345kh_hg@ukr.net O. Makove ichuk $\mathrm{PhD}$

Department of Electronic Computers*

M. Chomik

$\mathrm{PhD}$, Senior Research Center for Military and Strategic Studies***

V. Kh ud o v

$\mathrm{PhD}$, Junior Researcher Department of Information Technology Security*

I. K h i z h n y a k

$\mathrm{PhD}$, Lecturer

Department of Mathematical and Software Automated Control Systems**

V. Podlipaie v

$\mathrm{PhD}$, Researcher

Department of Information and Communication Technologies Institute of Telecommunications and Global Information Space

Chokolivskyi blvd., 13, Kyiv, Ukraine, 03186

Y. S heviakov

Doctor of Technical Sciences, Director Civil Aviation Institute**

O. B aranik

$\mathrm{PhD}$, Senior Lecturer Department of Aviation Armament Complexes**

A. I r k h a

$\mathrm{PhD}$, Senior Lecturer

Department of Space Systems and Geographic Information Support***

* Kharkiv National University of Radio Electronics Nauky ave., 14, Kharkiv, Ukraine, 61166 **Ivan Kozhedub Kharkiv National Air Force University Sumska str., $77 / 79$, Kharkiv, Ukraine, 61023 ***National Defense University of Ukraine named after Ivan Cherniakhovskyi

Povitroflotskyi ave., 28, Kyiv, Ukraine, 03049

Copyright (C) 2019, I. Ruban, H. Khudov, O. Makoveichuk, M.Chomik,

V. Khudov, I. Khizhnyak, V. Podlipaiev, Y. Sheviakov, O. Baranik, A. Irkha. This is an open access article under the CC BY license (http://creativecommons.org/licenses/by/4.0)
1. Introduction

At present, information technologies of tonal aerospace image processing are used in many fields: monitoring and evalua- tion of the environment; observing global changes; agriculture; extraction of minerals; monitoring renewable natural resources; meteorology; cartography; military activities, etc. [1-4]. Tonal digital aerospace images contain the most accurate and opera- 
tive information when compared with other sources [3]. These images are used to create the main component of geoinformation systems - electronic maps $[3,5]$. Electronic maps are the vector descriptions of spatial objects grouped into vector layers [5]. Constructing and updating electronic maps using tonal aerospace images is relevant for $[3,5,6]$ :

- management of enterprises infrastructure;

- optimization of transportation routes;

- construction planning;

- ecological monitoring, etc.

The main and most labor-intensive stage in making and updating of electronic maps with the use of tonal aerospace images is the construction and distribution of the vector representations of objects over thematic layers (aerospace images vectorization) $[3,5,7]$. The resulting set of thematic layers is the base of an electronic map, which is supplemented with the necessary geospatial and attributive information, or is a source of data to add missing objects. The vectorized images, when compared with raster ones, have the following advantages: scalability, less storage volume, high quality [7].

The main operation that affects quality of the tonal aerospace image vectorization is to define the objects' contours $[2,3,5,7]$. Known methods $[2,3,5,7]$ are used to determine the contours of objects. The basic known methods employ the criteria for a maximum first derivative's module and crossing zero of the second derivative in the direction of a brightness function gradient [8,9]. The formed contour image (a binary contour map) is encoded with the formation of a vector representation of the boundary lines of objects and details of their inner structure. The main disadvantages of known methods for determining contours are:

- the need to restore gaps in contours;

- the need to ensure grouping of individual contours into contour representations of objects;

- low quality in determining the contours of objects with a smooth drop of the brightness function at the border with a background;

- complexity of localizing a contour line's center due to the existence of the region of its location's uncertainty.

In addition, known methods for determining objects' contours do not take into consideration features of tonal aerospace images. Therefore, most known methods cannot be directly applied to determining the contours on tonal aerospace images.

Thus, it is a relevant task to develop new methods for determining the contours of objects on tonal aerospace images.

\section{Literature review and problem statement}

We shall briefly analyze known methods for determining the contours of objects on dissimilar images, including the aerospace images.

Paper [8] suggests methods to determine the contours of objects on images based on the use of a two-dimensional differential scalar Laplace operator. The main drawbacks are the impossibility to define the boundary direction, not the selection but rather the underlining of a brightness differential.

Article [9] proposed determining the boundaries by gradient methods, which calculate the full vector of an image gradient. The main disadvantages are the complexity of solving a Bayesian problem, the need for a priori knowledge of the conditionality of gradient values.
Study [9] applied various methods of spatial differentiation (methods by Sobel, Previt, Roberts, Wallace, sequential masking, etc.) to determine boundaries on an image. The main disadvantages of these methods are the presence of gaps, points and strokes, which form a noise background, the need to know the initial approximation to the sought limit and significant computational costs. The disadvantage of sequential masking methods is a reduced image contrast, blur. The disadvantage of the Laplacian-Gaussian method (LoG) [9] is the non-orientation of the Laplace and Gauss operators. Given this, the method is sensitive to a change in brightness along a parallel direction, which reduces the signal-noise ratio.

The Canny boundary selection method is used in [10] for identifying the contours on an image. The Canny method provides a high probability of detection, high accuracy of localization. A drawback of the Canny method is the disruption of boundaries at points of connections.

Paper [11] proposed a method for identifying enclosed paths in images based on the piecewise optimization strategy. The main drawback of method [11] is its applicability only for contours consisting of the Bezier curves.

The use of an active contour method is proposed in [12]. The disadvantages of the method are: a high accuracy of initial approximations, significant computational costs.

Study [13] proposes using neural networks for image vectorization and for determining contours. However, neural networks require an operator's participation, preprocessing of images; different initial conditions for image acquisition lead to different vectorization results. The neural network is trained on random selection, the final weight ratios for output neurons depend on the original sequence. Completion of the learning process is not based on rigorous optimization mathematical models. Thus, the neural network methods are not suitable for determining contours of objects on aerospace images.

Paper [14] suggests the use of neural networks for mapping and land cadaster applying images from the system World View-2 (DigitalGlobe company, the United States of America). The methods proposed in [14] solve the tasks in rural areas. The use of methods [14] is complicated for determining contours on aerospace images.

Methods reported in [15-17] are based on that objects consist of geometric primitives (straight lines, circumference, etc.). Work of such methods is based on the integral vector transformation by Radon [15] and the Huff's transformation [16]. The specified methods provide qualitative determination of geometric primitives in images from unmanned aircraft, for example, when an electric transmission line is detected in the forest terrain. The disadvantage of Radon transform [15] is its computational complexity. The number of mathematical operations for geometric primitives and complex-shape geometric objects is no different. The use of the Huff's transformation [17] is advisable in the case of defining geometric primitives in simple images, in terms of location of objects. These can be the images of forest arrays, agricultural fields, rivers, seas and oceans, etc. Under conditions of complex-structured aerospace images the Huff's transformation determines a large number of "junk" objects. This significantly influences quality of further vectorization and decryption of aerospace images.

When processing tonal medical images to determine geometric primitives, authors of [18] consistently apply methods of defining contours, boundaries, as well as the Huff's and Radon transformations. The results reported in [18] make it 
possible to qualitatively define geometric primitives on medical images. However, medical images [18] are substantially different from aerospace images in their structure.

Paper [19] uses an artificial bee colony method to thematically segment opto-electronic images from the onboard surveillance systems. The disadvantage of the method reported in [19] is a significant computing cost.

Study [20] builds on results from [19]. The authors stated an optimization problem on the segmentation of images from the onboard surveillance systems using an artificial bee colony method. The disadvantage of the method reported in [20] is the identification of areas that are potential objects rather than the objects' contours.

Paper [21] proposed an evolutionary method for thematic segmentation of images from the onboard surveillance systems. The method is based on ant algorithms. However, paper [21] examined only the test examples of the method execution; the fitness function has not been defined, etc. The main disadvantage of the method is the presence on the resulting image of a large number of "junk" objects.

Thus, the methods for determining objects' contours in the images have certain drawbacks and cannot be directly applied to defining objects' contours in aerospace images.

To undertake further research, we set the task of constructing methods for determining the contours of objects in tonal aerospace images based on ant algorithms. The main advantages of ant algorithms are the possibility of their effective division into parallel processes, adaptation, high performance, optimization of control, independence from poor initial decisions.

\section{The aim and objectives of the study}

The aim of this study is to construct methods for determining the contours of objects in tonal aerospace images based on ant algorithms.

To accomplish the aim, the following tasks have been set:

- to develop the basic principles and stages of the method for determining the contours of objects on a tonal aerospace image based on the ant algorithm;

- to process a tonal image in order to define the contours of objects by the method based on the ant algorithm;

- to devise the basic principles and stages of the method for multi-scale processing of aerospace images based on the ant algorithm;

- to carry out the multi-scale processing of tonal aerospace images with different scale values by methods based on the ant algorithms;

- to estimate the first and second kind errors in determining the contours of objects on tonal aerospace images based on the ant algorithms.

\section{Materials for constructing methods for determining the contours of objects on tonal aerospace images based on the ant algorithms}

4. 1. The main principles and stages of the method for determining the contours of objects on a tonal aerospace image

When constructing methods for determining the contours of objects on tonal aerospace images based on the ant algorithms, we shall consider the following assumptions and limitations:
- the source image $f(\mathbf{X})$, where $\mathbf{X}(x, y)$ are the coordinates of pixels on an image, is a tonal aerospace image;

- the number of brightness gradations of a tonal aerospace image is 256 ;

- a tonal aerospace image is considered after a pre-processing stage;

- an image is not distorted;

- a large number of dissimilar objects are present in an image;

- the objects are compact and low-contrast in comparison with the background.

The generalized description of the ant algorithm can be represented by expression (1) [22]:

$A C O=\{S, M 1, A, P, I n, O u t\}$

where $A C O$ - ant colony optimization (optimization by the method of an ant colony or the ant algorithm); $S$ - set of agents; $M 1$ - an object for the exchange of experience between agents (ants); $A$ - the rules of the ant algorithm performance (construction, behavior, modification of agents); $P$ - parameters used in rules $A$ (heuristic coefficients); In, Out - input and output of the ant algorithm (interaction with external environment and control system).

The scheme of relations between agents and the external environment is shown in Fig. 1.

In Fig. 1, we used the following designations:

$-I n=\left\{I_{1}, I n_{f b}\right\}$;

- $I n_{1}$ - inputs that receive the fitness function $\varphi\left(\mathbf{X}_{i j}\right)$ and constraints $\mathrm{G}(\mathbf{X})$;

$-\mathbf{X}(x, y)$ - vector of agents' positions;

- $\mathbf{X}_{i j}\left(x_{i j}, y_{i j}\right)$ - vector of agents' positions at the $j$-th iteration at the i-th pixel of an image;

- In $n_{f b}$ - input for a feedback;

- Out $=\left\{\mathrm{Out}_{1}, \mathrm{Out}_{f b}\right\}$

- Out 1 - outputs for the best-found solution $\mathbf{X}_{\text {final }}^{\text {best }}$ and the optimal value for fitness function $\varphi\left(\mathbf{X}_{\text {final }}^{\text {best }}\right)$;

- Out $f b$ - output for a feedback;

- $U_{c i}-$ controlling influence (start, stop, number of agents, method's parameters, etc.).

The scheme of the method for defining the contours of objects on an aerospace image based on the ant algorithm is shown in Fig. 2. The scheme represents the sequence of method's actions:

1. Initialize the starting locations of agents on the image in the first iteration $(j=1) . \mathbf{X}_{i 1}\left(x_{i 1}, y_{i 1}\right)$ - vector of agents' positions in the first iteration, $i=1,2, \ldots, S ; S$ - total number of agents. The total number of agents $S$ equals the number of pixels in the original image.

2. Calculate fitness function $\varphi_{j}(\mathbf{X})$ in the $j$-th iteration. The fitness function to be defined in the $j$-th iteration is function (2):

$$
\varphi_{j}(X)=\sum_{m=1}^{S} \sum_{i=1}^{N}\left(P_{i}^{m}(j) D_{i}^{m}(j)\right)
$$

where $m$ is the current number of an agent; $N$ - image size; $P_{i}^{m}(j)$ - probability of the $m$-th agent transition to the $i$-th turning point of the route in the $j$-th iteration (3):

$$
P_{i}^{m}(j)=\frac{\left(F_{i}^{m}(j)\right)^{\alpha}\left(L_{i}^{m}(j)\right)^{\beta}}{\sum_{r=1}^{R}\left(F_{r}^{\alpha}(j) \cdot L_{r}^{\beta}(j)\right)},
$$


where $\alpha$ and $\beta$ are the parameters that set the weight of pheromone and "greed" of the method, respectively;

$-R$ - number of possible turning points along a route;

$-L_{i}^{m}(j)$ is the attractiveness of the route's section to the $m$-th agent at the $i$-th point of the image in the $j$-th iteration;

- $F_{i}^{m}(j)$ is the concentration of the $m$-th agent's pheromone at the $i$-th point of the image in the $j$-th iteration;

- function $D_{i}^{m}(j)$ defines the length of the route's section, taking into consideration the difference in the brightness of neighboring pixels for the $m$-th agent at the $i$-th point of the image in the $j$-th iteration; it is determined from expression (4):

$$
D_{i}^{m}(j)=\left|\Delta x_{i}^{m}(j)\right|+\left|\Delta y_{i}^{m}(j)\right|+k\left|\Delta f_{i}^{m}(j)\right|,
$$

where $\left|\Delta x_{i}^{m}(j)\right|, \quad\left|\Delta y_{i}^{m}(j)\right|$ are the elementary displacements of the $m$-th agent at the $i$-th point of the image in the $j$-th iteration along the axes $x$ and $y$, respectively;

$-k$ - coefficient that takes into consideration the difference in scale along the $x$ and $y$ axes and brightness of image pixels and different units of measurement of elementary displacements and brightness. If brightness accepts a value from range [0..255], then $k=1$;

- $\Delta f_{i}^{m}(j) \mid$ is the difference in the brightness of neighboring pixels for the $m$-th agent at the $i$-th point of the image in the $j$-th iteration $-(5)$ :

$$
\begin{aligned}
& \left|\Delta f_{i}^{m}(j)\right|= \\
& =\left|f\left(x_{i}^{m}(\mathrm{j}), y_{i}^{m}(\mathrm{j})\right)-f\left(x_{i-1}^{m}(\mathrm{j}), y_{i-1}^{m}(\mathrm{j})\right)\right| .
\end{aligned}
$$

3. Movement of agents. In an ant algorithm, during each iteration of the iterative process, $m$ agents search for a solution and for the pheromone renewal along the found route. Each $m$-th agent, when segmenting an image, starts the way from the initial point of the route, sequentially passing the turning points along the route selected by the method, and completes the way at one of the end points of the route. The movement of the agents is based on the criterion for a minimum of fitness function (2) which, taking into consideration the four-connectedness of the movement of agents (6):

$$
\left|\Delta x_{i}^{m}(j)\right|+\left|\Delta y_{i}^{m}(j)\right|=1,
$$

takes the form (7):

$$
\begin{aligned}
& \varphi_{j}(X)= \\
& =\sum_{m=1}^{S} \sum_{i=1}^{N}\left(P_{i}^{m}(j)\left(1+k\left|\begin{array}{l}
f\left(x_{i}^{m}(j), y_{i}^{m}(j)\right)- \\
-f\left(x_{i-1}^{m}(j), y_{i-1}^{m}(j)\right)
\end{array}\right|\right)\right) \rightarrow \text { min. }
\end{aligned}
$$

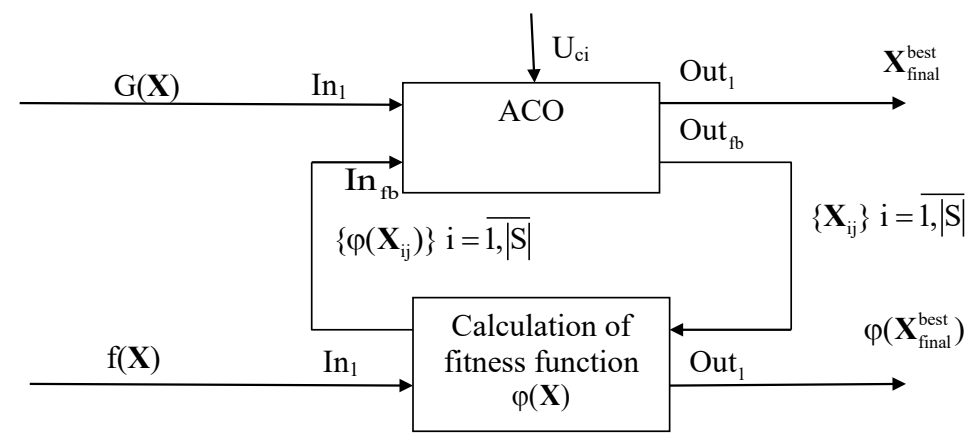

Fig. 1. Scheme of relations between agents and the external environment

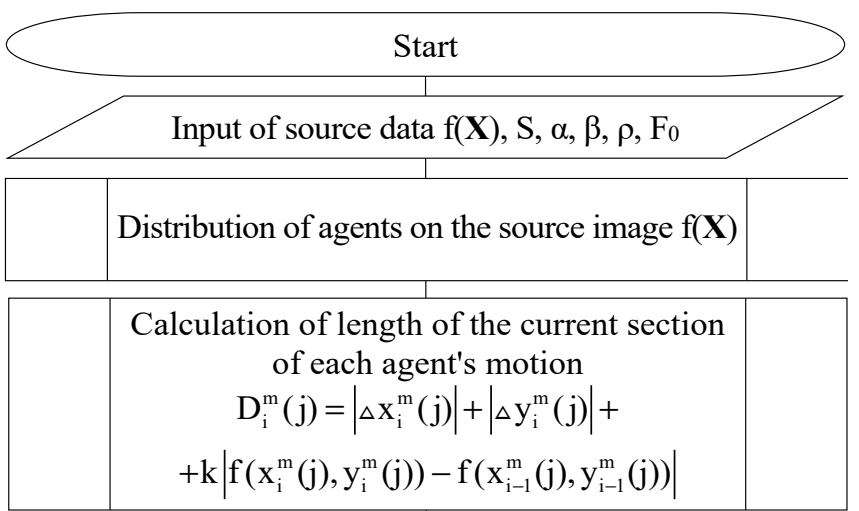

Calculation of route attractiveness

\begin{tabular}{|c|c|c|}
\hline Calculation of route attractiveness & \\
$\qquad \mathrm{L}_{\mathrm{i}}^{\mathrm{m}}(\mathrm{j})=\frac{1}{1+\mathrm{e}^{\frac{\mathrm{D}_{\mathrm{i}}^{\mathrm{m}}(\mathrm{j})}{\mathrm{D}_{0}}}}$ & \\
\hline
\end{tabular}

\begin{tabular}{|l|l|l|}
\hline Pheromone concentration renewal & \\
$\mathrm{F}_{\mathrm{i}}^{\mathrm{m}}(\mathrm{j}+1)=(1-\rho) \mathrm{F}_{\mathrm{i}}^{\mathrm{m}}(\mathrm{j})+\sum_{\mathrm{m}=1}^{\mathrm{M}} \Delta \mathrm{F}_{\mathrm{i}}^{\mathrm{m}}$ & \\
\hline
\end{tabular}

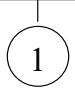

a

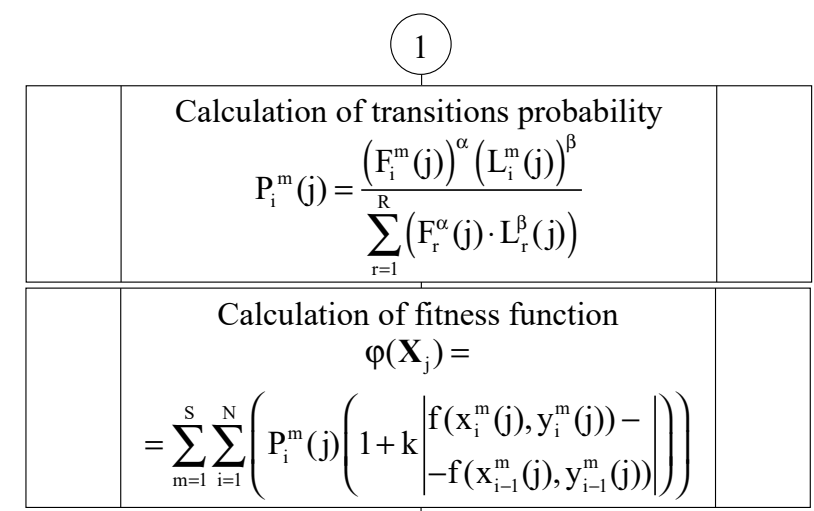

Checking the rule for terminating the method execution (minimal value for fitness function, boundary quantity of iterations $\mathrm{N}_{\text {iter }}$

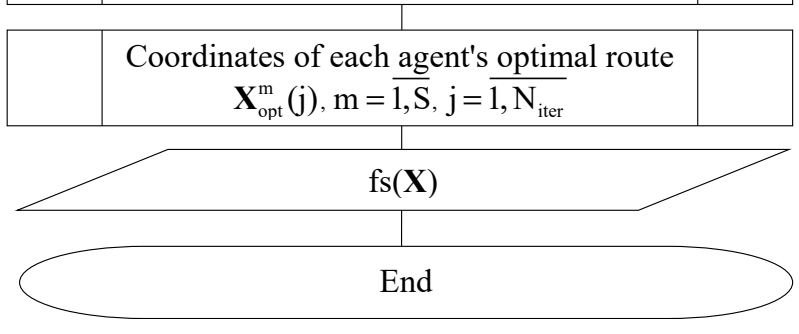

$b$

Fig. 2. Scheme of the method for determining the contours of objects on a tonal aerospace image based on the ant algorithm: $a$ - sheet $1 ; b$ - sheet 2 
We believe that the attractiveness of route's section $L_{i}^{m}(j)$ for the $m$-th agent at the $i$-th point of the image in the $j$-th iteration is inversely dependent on the length of the route's section, for example (8) to (10):

$$
\begin{aligned}
& L_{i}^{m}(j)=\frac{1}{D_{i}^{m}(j)}, \\
& L_{i}^{m}(j)=\frac{1}{1+\frac{D_{i}^{m}(j)}{D_{0}}}, \\
& L_{i}^{m}(j)=\frac{1}{1+e^{\frac{D_{i}^{m}(j)}{D_{0}}}},
\end{aligned}
$$

where $D_{0}$ is the parameter that takes into consideration a scale of the image.

At the beginning of the iterative process, the amount of pheromone along the itinerary is taken to be the same and equal to some small number $F_{0}$. After each iteration, the concentration of pheromone along the sections selected by agents is renewed in line with rule (11):

$$
F_{i}^{m}(j+1)=(1-\rho) F_{i}^{m}(j)+\sum_{m=1}^{M} \Delta F_{i}^{m}
$$

where $\rho \in[0,1]$ is the rate of evaporation of pheromone; $\Delta F_{i}^{m}$ is the concentration of pheromone along the $i$-th section of the route, formed by the passage of the $m$-th agent.

The result of a certain number of iterations is the determined, most attractive routes, based on the chosen criterion, with the maximum pheromone concentration. Pheromone gradually evaporates along the unattractive routes, thereby eliminating such routes. At $\alpha=0$, agents at each step move to the nearest turning point of the route, and the ant algorithm is transformed into a "greedy" method from a classical optimization theory. At $\beta=0$, only the effect of pheromones is taken into consideration, which could quickly lead to a suboptimal solution.

4. Check the termination conditions. If the condition is met, the original image $f s(\mathbf{X})$ that has the defined objects' contours is retrieved. Otherwise, proceed to point 2 .

The parameters of $P(1)$ for the method are determined as $P\left(\alpha, \beta, \rho, F_{0}\right)$.

Thus, the method of determining the contours of objects on a tonal aerospace image based on the ant algorithm, in contrast to known ones:

- takes into consideration patterns of image formation;

- employs the ant algorithm to determine the contours;

- reduces determining the contours of objects on an image to the calculation of the fitness function, the totality of agents' traffic areas and the pheromone concentration along the agents' traffic routes.

4.2. Processing of a tonal image to determine the contours of objects by the method based on the ant algorithm

The initial tonal images that we shall consider are images from the spacecraft WorldView-1 (DigitalGlobe company, the United States of America) (Fig. 3) [23]. The images are an area of the cruising liner Costa Concordia disaster (Fig. 3, a) and an image of the city of San Francisco (the United States of America) (Fig. 3, b). The images are represented in tonal gradations in the grayscale range from 0 to 255 . Dimensions of the images in Fig. 3 are $(640 \times 640)$ pixels.

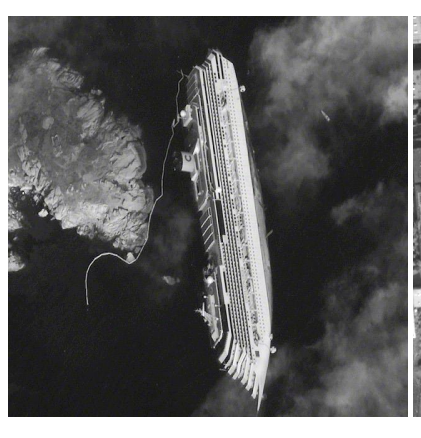

$a$

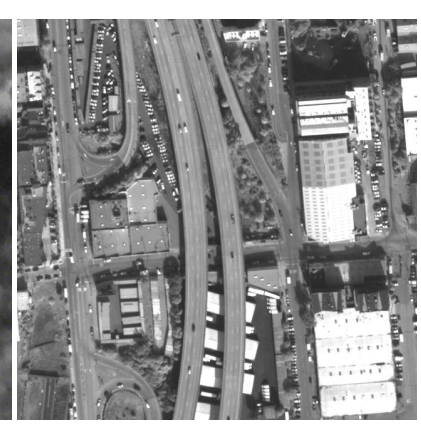

$b$
Fig. 3. Initial tonal images: $a$ - region of the cruising liner Costa Concordia disaster; $b$-image of the city of San Francisco (the United States of America) [23]

Parameters of the method for thematic segmentation of tonal images based on the ant algorithm are constant at each iteration and are equal to: $S=409,600$ agents; $\alpha=2 ; \beta=1$; $\rho=10^{-3} ; F_{0}=10^{-2}$.

The calculations were performed by programming using a high-level programming language and an interactive environment for programming, numerical calculations and visualization of results employing MATLAB R2017b.

The condition for terminating the iterative process of determining the contours on tonal images based on the ant algorithm is the boundary number of iterations (50 iterations).

Results from processing the initial tonal images (Fig. 3, $a, b$ ) to determine the contours of objects by the method based on the ant algorithm are shown in Fig. 4.

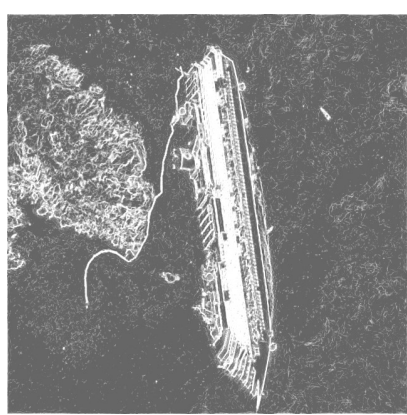

$a$

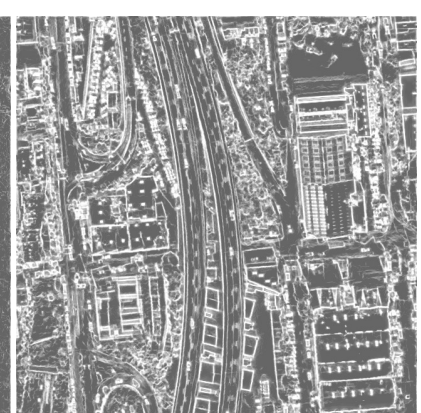

$b$
Fig. 4. Initial images with the contours determined by the method based on the ant algorithm: $a-$ area of the cruising liner Costa Concordia disaster; $b$-image of the city of San Francisco (the United States of America)

Fig. 4 shows that the visual assessment of the quality of contours determination makes it possible to define the objects - the state of the damage to the liner, the objects of urban infrastructure, transport, etc. It is necessary to emphasize the presence on the image (Fig. 4) of a large number of highlighted contours of small-size objects, the so-called "junk" objects. In order to reduce the number of "junk" objects in the resulting image, we shall consider the basic principles and stages of the method for multiscale processing of aerospace images based on the ant algorithm. 
4.3. Basic principles and stages of the method for multi-scale processing of aerospace images based on the ant algorithm

The essence of the multiscale conversion of aerospace images is illustrated in Fig. 5.

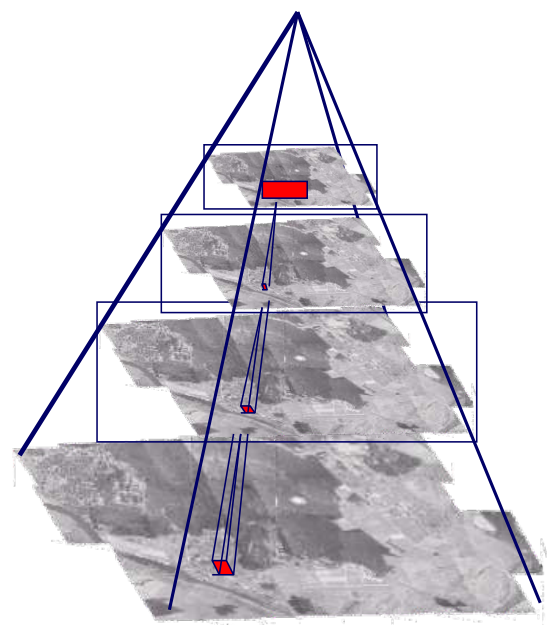

Fig. 5. Essence of multiscale conversion of aerospace images

The multiscale conversion of initial image $f(x, y)$, where $(x, y)$ are the spatial coordinates of the image, shall be represented in form (12) [24]:

$$
L\left(x, y, K_{m}\right)=g\left(x, y, K_{m}\right) * f(x, y),
$$

where $L\left(x, y, K_{m}\right)$ is the multi-scale conversion of initial image $f(x, y) ; g\left(x, y, K_{m}\right)$ is the conversion core; $K_{m}$ is a scale factor; * is the convolution operator.

The core of the conversion $g\left(x, y, K_{m}\right)$ is chosen in the form of a Gaussian (13) (Fig. 6):

$$
g\left(x, y, K_{m}\right)=\frac{1}{2 \pi \sqrt{K_{m}}} e^{-\frac{\left(x^{2}+y^{2}\right)}{2 K_{m}}} .
$$

In expression (12) the convolution is performed based on spatial coordinates $(x, y)$, and the scaling factor $K_{m}$ indicates only the scale of the convolution operation. The choice of such a core of the conversion is due to the uniqueness of a Gaussian nucleus, which includes linearity, invariance to the shift, to scale distortions, to image rotation and to not enhancing local extrema in the original image [24].

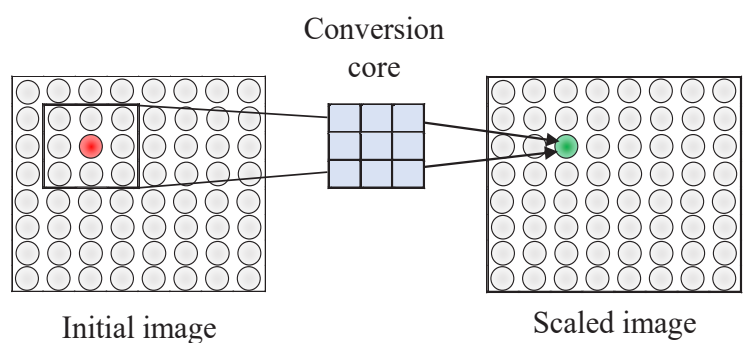

Fig. 6 . The principle of scaled image acquisition

The scaling factor $K_{m}$ serves as the variance in expression (13) for a Gaussian. At $K_{m}=0$, the conversion core $g(x, y, 0)$ becomes a pulse function, such that $L(x, y, 0)=f(x, y)$, that is the scale conversion of the original image is precisely the original image $f(x, y)$. When the scale factor $K_{m}$ is increased, $L\left(x, y, K_{m}\right)$ is the result of smoothing the initial image $f(x, y)$.

The improvement of quality of multiscale processing of aerospace images is based on that the objects are on images with a different scale value $K_{m}$. In this case, the decrypting features of objects can manifest themselves to a certain degree in the images of multiple scales. In addition, some of the decrypted features of the objects may change so that it is able to be considered as another (additional) de-encrypting attribute.

The scheme of the method for multi-scale processing of aerospace images based on the ant algorithm is shown in a general form in Fig. 7. The essence of the method for multiscale processing of aerospace images based on the ant algorithm comes down to the following.

1. Enter source data, namely (Fig 7$): \mathbf{f}_{K_{m}}(\mathbf{X})$ - aerospace image of scale $K_{m}$.

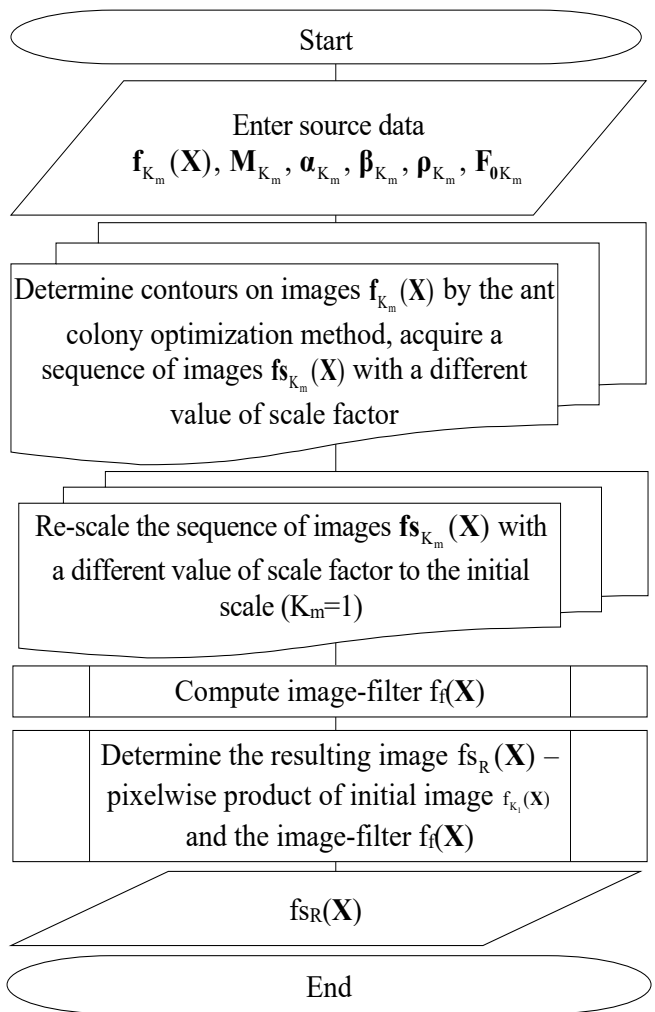

Fig. 7. Scheme of the method for multi-scale processing of tonal aerospace images based on the ant algorithm

In Fig. 7:

$-\mathbf{X}(x, y)$ - vector of agents' positions on the image of scale $K_{m}$

$-\mathbf{M}_{K_{m}}$ - number of agents on the image of scale $K_{m}$;

$-\boldsymbol{\alpha}_{K_{m}}$-weight of pheromone on the image of scale $K_{m}$;

- $\boldsymbol{\beta}_{K_{m}}$ - "greed" of the method on the image of scale $K_{m}$;

$-\boldsymbol{\rho}_{K}$ - rate of pheromone evaporation on the image of scale $K_{m}^{m}$

$-\mathbf{F}_{K_{m}}$ - starting amount of pheromone on the image of scale $K_{m}$

2. Determine contours on images $\mathbf{f}_{K}(\mathbf{X})$ by the method based on the ant algorithm, acquire a sequence of images $\mathbf{f s}_{K}(\mathbf{X})$ with different scale factor values.

3. Rescale the sequence of images $\mathbf{f s}_{K_{m}}(\mathbf{X})$ with different scale-factor values to the original scale $\left(K_{m}=1\right)$. 
4. Compute the image-filter $f_{f}(\mathbf{X})$. In this case, the brightness of each pixel on the image-filter is determined based on the image informativeness metric with different scale values and is calculated by averaging the brightness of the corresponding pixels of images of each scale (expression (14)):

$$
f_{f}(\mathbf{X})=\frac{\sum_{m=1}^{N} f s_{K_{m}}(\mathbf{X})}{N},
$$

where $N$ is the number of scaled images.

5. Determine the resulting image $f_{R}(\mathbf{X})$ as a pixelwise product of the source image $f_{K_{1}}(\mathbf{X})$ and the image-filter $f_{f}(\mathbf{X})$ (expression 15):

$$
f s_{R}(\mathbf{X})=f_{K_{1}}(\mathbf{X}) \& f_{f}(\mathbf{X}),
$$

where \& is the logical operation "conjunction" ("logical multiplication").

Table 1 is the truth table of the logical operation "conjunction" for the resulting image $f s_{R}(\mathbf{X})$.

Table 1

Truth table of the logical operation "conjunction" for the resulting segmented image $f s_{R}(\mathbf{X})$

\begin{tabular}{|c|c|c|}
\hline$f_{K_{1}}(\mathbf{X})$ & $f_{f}(\mathbf{X})$ & $f s_{R}(\mathbf{X})$ \\
\hline 1 & 1 & 1 \\
\hline 0 & 1 & 0 \\
\hline 1 & 0 & 0 \\
\hline 0 & 0 & 0 \\
\hline
\end{tabular}

Thus, the resulting image $f s_{R}(\mathbf{X})$ would contain the objects' pixels only if the pixels are present on all images with a different scale value.

6. Output the resulting image $f s_{R}(\mathbf{X})$.

Therefore, the method for multi-scale processing of aerospace images based on the ant algorithm, in contrast to known ones, implies the following:

- determining the contours on images with a different value of scale factor using the method based on the ant algorithm;

- rescaling of images with a different value of scale factor to the initial size and computation of the image-filter;

- the resulting image is a pixelwise product of the original image and the image-filter.

4. 4. Multiscale processing of tonal aerospace images with different scale values using methods based on the ant algorithms

The original images to be considered are the tonal images from the spacecraft WorldView-1 (DigitalGlobe company, the United States of America) (Fig. 3) [23] with different scale values $K_{m}=1,2,3, \ldots, 31,32$. Multiscale processing of the tonal images will be carried out by the method of multi-scale processing of tonal aerospace images based on the ant algorithm (Fig. 7). The method parameters are permanent for images with different scale factor values at each iteration and are equal to: $\mathbf{M}_{K_{m}}$ equals the number of pixels on the image of the corresponding scale; $\boldsymbol{\alpha}_{K_{m}}=2 \mathbf{I} ; \boldsymbol{\beta}_{K_{m}}=\mathbf{I}$; $\boldsymbol{\rho}_{K_{m}}=10^{-3} \mathbf{I} ; \quad \mathbf{F}_{0 K_{m}}=10^{-2} \mathbf{I} ; \mathbf{I}$ is the unity matrix. The method parameters were determined by selecting and were not optimized. Optimization of the specified parameters is a separate scientific task and is the subject of further research.
The tonal images with defined contours at different scale values and rescaled to scale $K_{m}=1$ are shown in Fig. 8 . The condition to terminate the method, based on the ant algorithm, for determining the contours of images at each scale is the limit of iterations equal to 50 .

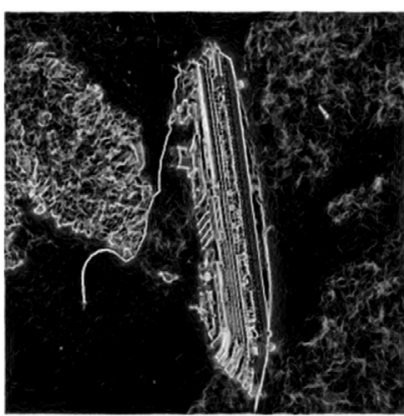

a

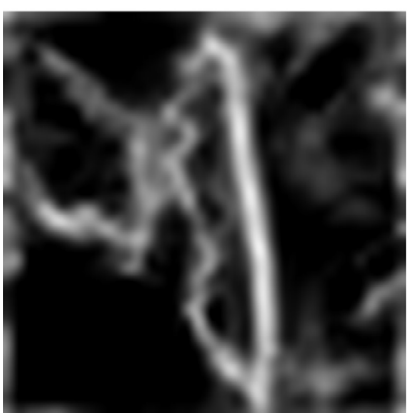

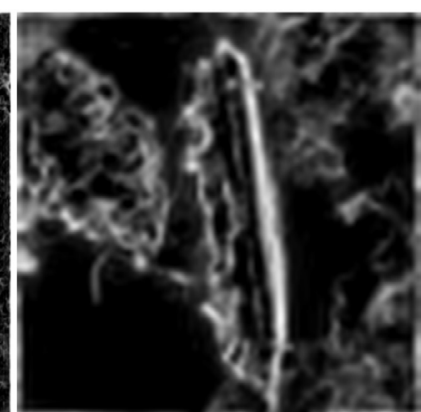

$b$

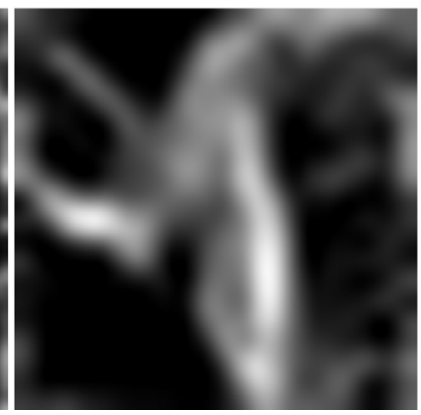

d
Fig. 8. Tonal images with defined contours at different scale values and rescaled to scale $K_{m}=1: a-K_{m}=2 ; b-K_{m}=8$;

$$
c-K_{m}=16 ; d-K_{m}=32
$$

The image-filter $f_{f}(\mathbf{X})$ is calculated from expression (14) and is shown in Fig. 9. The brightness of each pixel in the image-filter is defined by the image informativeness metric at different scale values and is calculated by averaging the brightness of the corresponding pixels of images with different scale values. The resulting image $f s_{R}(\mathbf{X})$ is determined from expression (15) as a pixelwise product of the source image $f_{K_{1}}(\mathbf{X})$ and the image-filter $f_{f}(\mathbf{X})$ and is shown in Fig. 10. Calculations were performed using the high-level programming language and interactive environment for programming, numerical calculations and visualization of results employing MATLAB R2017b. By comparing Fig. 4, $a$ and Fig. 10 we have established that the use of multi-scale processing reduces the number of "junk" objects. At the same time, due to the multi-scale processing in Fig. 10, unlike in Fig. 4, $a$, not the objects' contours are defined but the objects in full.

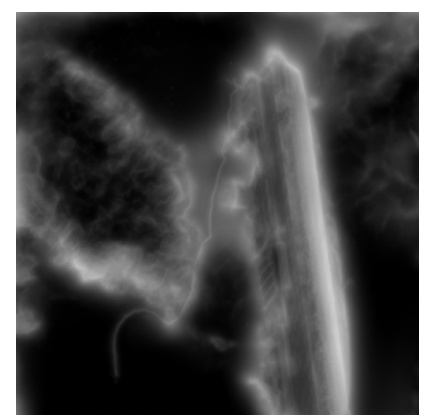

Fig. 9. Image-filter $f_{f}(\mathbf{X})$ 


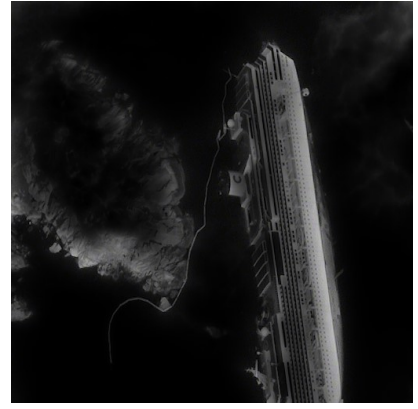

Fig. 10. Resulting image $f s_{R}(\mathbf{X})$

Consider another original image (Fig. 3, b) - image of the city of San Francisco from the spacecraft WorldView-1. The tonal images with defined contours at different scale values and rescaled to the scale $K_{m}=1$ are shown in Fig. 11 . The image-filter $f_{f}(\mathbf{X})$ is shown in Fig. 12. The resulting image $f s_{R}(\mathbf{X})$ is shown in Fig. 13.
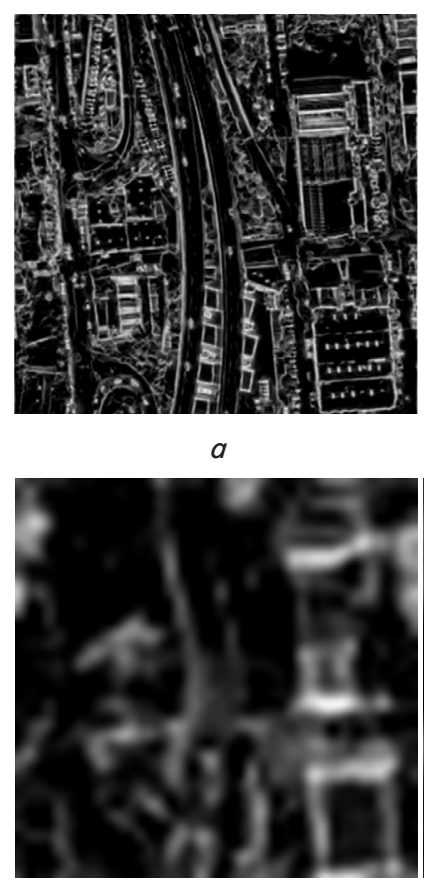

c

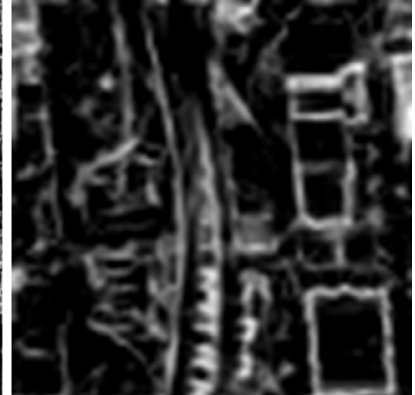

$b$

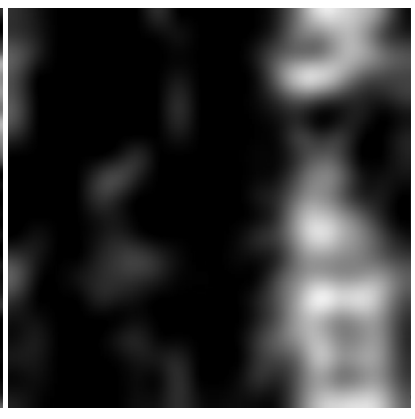

$d$
Fig. 11. Tonal images with defined contours at different scale values and rescaled to the scale $K_{m}=1: a-K_{m}=2 ; b-K_{m}=8$; $c-K_{m}=16 ; d-K_{m}=32$

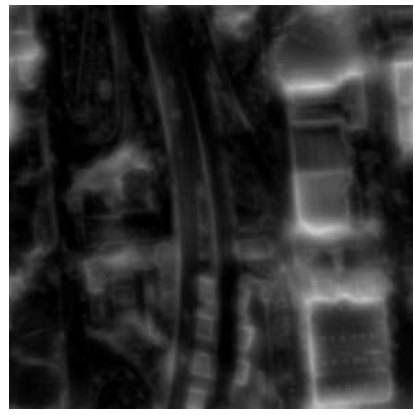

Fig. 12. Image-filter $f_{f}(\mathbf{X})$

Calculations were conducted using the high-level programming language and interactive environment for programming, numerical calculations and visualization of re- sults MATLAB R2017b. By comparing Fig. 4, $b$ and Fig. 13 we have found that the use of multi-scale processing reduces the number of "junk" objects. At the same time, due to the multi-scale processing in Fig. 13, unlike Fig. 4, $b$, not the objects' contours are defined but the objects in full.

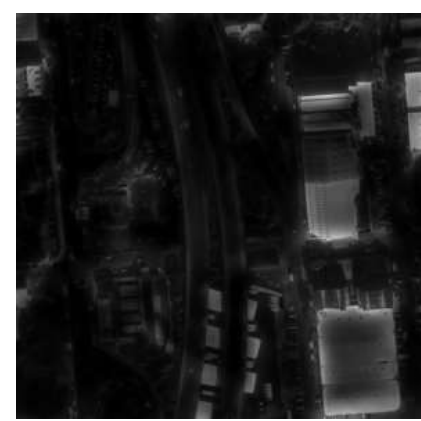

Fig. 13. Resulting image $f s_{R}(\mathbf{X})$

4. 5. Estimation of the first and second kind errors in determining the contours of objects on tonal aerospace images based on the ant algorithms

The indicators for determining the quality of contours of objects on tonal aerospace images shall be errors of first and second kind. The errors in determining objects of urban infrastructure of first $\left(\alpha_{1}\right)$ and second $\left(\beta_{2}\right)$ kind are determined based on the maximum likelihood criterion, which follows from the generalized criterion of an average risk minimum [20]. The errors in determining elements of urban infrastructure of first kind $\alpha_{1}$ and second kind $\beta_{2}$ are calculated from expressions (16), (17), respectively [20]:

$$
\begin{aligned}
& \alpha_{1}=\frac{S_{1}\left(f_{s}(\mathbf{X})\right)}{S_{2}(f(\mathbf{X}))}, \\
& \beta_{2}=1-\frac{S_{3}(f s(\mathbf{X}))}{S_{4}((f(\mathbf{X}))},
\end{aligned}
$$

where $S_{1}\left(f_{s}(\mathbf{X})\right)$ is the area of plots (number of pixels) of the background that is mistakenly attributed to the objects' contours on image $f_{s}(\mathbf{X})$;

$-S_{2}(f(\mathbf{X}))$ is the area of plots (number of pixels) in the background of the source image $f(\mathbf{X})$;

$-S_{3}\left(f_{s}(\mathbf{X})\right)$ is the plane (number of pixels) of correctly defined objects' contours on image $f_{s}(\mathbf{X})$;

$-S_{4}(f(\mathbf{X}))$ is the plane (number of pixels) of the contours of objects on the original image $f(\mathbf{X})$.

Calculations based on expressions (16), (17) shall be carried out under the same conditions, the same signal/noise ratio for the methods of determining the contours of objects employed by known methods. The chosen known methods are the Canny method and a Random forest method. Comparison shall be carried out using the method of determining the contours of objects on a single aerospace image based on the ant algorithm and applying the method of multi-scale processing of aerospace images based on the ant algorithm.

The values for first and second kind errors for different methods, calculated from expressions (16), (17), are given in Table 2 .

Analysis of data from Table 2 indicates a reduction in the first and second kind errors in determining contours on tonal aerospace images when using the constructed methods. The errors of determining the contours of objects on tonal aerospace images decreased on average by the magnitude of $18-22 \%$. 
Table 2

Estimation of the first and second kind errors in determining the contours of objects on tonal aerospace images by different methods

\begin{tabular}{|c|c|c|c|}
\hline \multicolumn{2}{|c|}{ Methods for determining objects' contours } & $\alpha_{1}, \%$ & $\beta_{2}, \%$ \\
\hline \multirow{2}{*}{ Canny } & $\begin{array}{c}\text { Image of the region of the } \\
\text { cruising liner Costa Con- } \\
\text { cordia disaster (Fig. 3, } a \text { ) }\end{array}$ & 23 & 26 \\
\cline { 2 - 5 } & $\begin{array}{c}\text { Image of the city of San } \\
\text { Francisco (Fig. 3, } b \text { ) }\end{array}$ & 27 & 30 \\
\hline Random forest & $\begin{array}{c}\text { Image of the region of the } \\
\text { cruising liner Costa Con- } \\
\text { cordia disaster (Fig. 3, } a \text { ) }\end{array}$ & 27 & 31 \\
\cline { 2 - 5 } & $\begin{array}{c}\text { Image of the city of San } \\
\text { Francisco (Fig. 3, } b \text { ) }\end{array}$ & 32 & 36 \\
\hline $\begin{array}{c}\text { Method for determining } \\
\text { the contours of objects } \\
\text { on a single aerospace } \\
\text { image based on the ant } \\
\text { algorithm (Fig. 2) }\end{array}$ & $\begin{array}{c}\text { Image of the region of the } \\
\text { cruising liner Costa Con- } \\
\text { cordia disaster (Fig. 3, } a \text { ) }\end{array}$ & 13 & 17 \\
\cline { 2 - 5 } & $\begin{array}{c}\text { Image of the city of San } \\
\text { Francisco (Fig. 3, } b \text { ) }\end{array}$ & 17 & 21 \\
\hline $\begin{array}{c}\text { Method of multi-scale } \\
\text { processing of aerospace } \\
\text { images based on the ant } \\
\text { algorithm (Fig. 7), } \\
K_{m}=1,2,3, . ., 31,32\end{array}$ & $\begin{array}{c}\text { Image of the region of the } \\
\text { cruising liner Costa Con- } \\
\text { cordia disaster (Fig. 3, } a \text { ) }\end{array}$ & 6 & 10 \\
\cline { 2 - 5 } & $\begin{array}{c}\text { Image of the city of San } \\
\text { Francisco (Fig. 3, } b \text { ) }\end{array}$ & 10 & 13 \\
\hline
\end{tabular}

\section{Discussion of results of constructing the method for determining the contours of objects on tonal aerospace images based on the ant algorithms}

We have devised the basic principles and stages of the method for determining the contours of objects on tonal aerospace images based on the ant algorithms. It was established that the main stages of the method are (Fig. 2): to initialize the starting positions of agents on an image during first iteration; calculate the fitness function; move agents; check the condition for terminating the method execution. A special feature of fitness function (7) is that at the same time the brightness of image pixels is taken into consideration, the weight of pheromones, the attractiveness of agents' route's section considering the four-connectedness of agents' moves (6). The method for determining contours of objects on a tonal aerospace image is based on the ant algorithm; in contrast to known ones, it takes into consideration patterns in the image formation. Determining the contours employs an ant algorithm. Determining the objects' contours on an image is reduced to calculating the fitness function, the totality of agents' motion sections and the pheromone concentration along the agents' motion routes.

We have processed tonal images (Fig. 3, $a, b$ ) to determine the contours of objects by the method based on the ant algorithm. It was established that the visual estimation of quality of determining the contours (Fig. 4, $a, b$ ) makes it possible to define objects - the state of the damage to the sea liner, the objects of urban infrastructure, transport etc. We note that there is a large number of selected contours of small-size objects ("junk" objects) in the resulting image.

In order to reduce the number of "junk" objects on the resulting image, the main principles and stages of the method of multi-scale processing of aerospace images based on the ant algorithm (Fig. 7) have been developed. The improvement of the quality of multiscale processing of aerospace images is based on that the objects are to be found on images with a different value of scale factor $K_{m}$. In this case, the decrypting features of objects can manifest themselves to a certain degree on images at multiple scales. In addition, some of the decrypted features of objects may change so that they shall be considered as another (additional) de-encrypting attribute. The main stages of the method of multi-scale processing of aerospace images based on the ant algorithm are (Fig. 7):

- enter source data;

- determine contours by the method based on the ant algorithm, acquire a sequence of images with different scale values;

- rescale the sequence of images with a different value for scale-factor to the original scale;

- compute the image-filter;

- determine the resultant image; output of the resulting image.

The multiscale processing of tonal aerospace images with different scale values was performed by using methods based on the ant algorithms. It was determined that the use of multi-scale processing reduces the number of "junk" objects. At the same time, due to the multi-scale processing, not only the contours of objects are determined but the objects in full (Fig. 10, 13).

We have estimated the first and second kind errors in determining the contours of objects on tonal aerospace images based on the ant algorithms. It was established that using the constructed methods reduces the first and second kind errors in determining contours on tonal aerospace images on average by the magnitude of $18-22 \%$ (Table 2 ).

The main disadvantage of the devised methods for determining the contours of objects on aerospace images is the need for a significant computing resource.

When undertaking the further research, it is necessary:

- to build a method for determining the contours of objects on colored aerospace images;

- to construct a method for multi-scale processing of colored aerospace images.

\section{Conclusions}

1. We have devised the main principles and stages of the method for determining the contours of objects on tonal aerospace images based on the ant algorithms. The method for determining the contours of objects on an image is reduced to calculating the fitness function, the totality of agents' motion sections and the pheromone concentration along the routes of agents' motion.

2 . The processing of a tonal image to determine objects' contours was performed using the method based on the ant algorithm. It was established that visual assessment of the quality of contour detection makes it possible to define objects. At the same time, a large number of "junk" objects are present on the resulting image.

3. We have devised the main principles and stages of the method for multi-scale processing of aerospace images based on the ant algorithm. The method of multi-scale processing of aerospace images based on the ant algorithm, in contrast to known ones, implies determining the contours on images with a different value of the scale factor by the method based on the ant algorithm. Additionally, one re-scales images with different scale factor values to the original size and computes the image-filter. The resulting image is a pixelwise product of the original image and the image-filter. 
4. The multiscale processing of tonal aerospace images with different scale values was performed using methods based on the ant algorithms. It was established that the use of multi-scale processing reduces the number of "junk" objects. At the same time, due to multi-scale processing, not the objects' contours are defined but the objects in full.
5. We have estimated the first and second kind errors in determining the contours of objects on tonal aerospace images based on the ant algorithms. It was established that using the constructed methods reduces the first and second kind errors in determining contours on tonal aerospace images on average by the magnitude of $18-22 \%$.

\section{References}

1. Weng, Q. (2009). Remote Sensing and GIS Integration. New York: McGraw-Hill Professional, 416.

2. Chemin, Y. (Ed.) (2012). Remote Sensing of Planet Earth. Rijeka, 250. doi: https://doi.org/10.5772/2291

3. Richards, J. (2013). Remote Sensing Digital Image Analysis. An Introduction. Springer. doi: https://doi.org/10.1007/978-3-642-30062-2

4. Vysotska, V., Lytvyn, V., Burov, Y., Gozhyj, A., Makara, S. (2018). The consolidated information web-resource about pharmacy networks in city. CEUR Workshop Proceedings (Computational linguistics and intelligent systems), 2255, 239-255.

5. Stryzhak, O., Prychodniuk, V., Podlipaiev, V. (2019). Model of Transdisciplinary Representation of GEOspatial Information. Advances in Information and Communication Technologies, 34-75. doi: https://doi.org/10.1007/978-3-030-16770-7_3

6. Lytvyn, V., Vysotska, V. (2015). Designing architecture of electronic content commerce system. 2015 Xth International Scientific and Technical Conference “Computer Sciences and Information Technologies” (CSIT). doi: https://doi.org/10.1109/stc-csit.2015.7325446

7. Karamti, H., Tmar, M., Gargouri, F. (2017). A new vector space model for image retrieval. Procedia Computer Science, 112, 771-779. doi: https://doi.org/10.1016/j.procs.2017.08.202

8. Gonzalez R. C., Woods R. E. (2017). Digital Image Processing. Prentice Hall, 1192.

9. Gupta, V., Singh, D., Sharma, P. (2016). Image Segmentation Using Various Edge Detection Operators: A Comparative Study. International Journal of Innovative Research in Computer and Communication Engineering, 4 (8), $14819-14824$.

10. Kabade, A., Sangam, V. (2016). Canny edge detection algorithm. International Journal of Advanced Research in Electronics and Communication Engineering (IJARECE), 5 (5), 1292-1295.

11. Yang, M., Chao, H., Zhang, C., Guo, J., Yuan, L., Sun, J. (2016). Effective Clipart Image Vectorization through Direct Optimization of Bezigons. IEEE Transactions on Visualization and Computer Graphics, 22 (2), 1063-1075. doi: https://doi.org/10.1109/ tvcg.2015.2440273

12. Sum, K., S. Cheung, P. (2006). A Fast Parametric Snake Model with Enhanced Concave Object Extraction Capability. 2006 IEEE International Symposium on Signal Processing and Information Technology. doi: https://doi.org/10.1109/isspit.2006.270844

13. Karamti, H., Tmar, M., Gargouri, F. (2014). Vectorization of Content-based Image Retrieval Process Using Neural Network. Proceedings of the 16th International Conference on Enterprise Information Systems, 435-439. doi: https://doi.org/10.5220/0004972004350439

14. Nyandwi, E., Koeva, M., Kohli, D., Bennett, R. (2019). Comparing Human Versus Machine-Driven Cadastral Boundary Feature Extraction. Remote Sens, 11, 1662. doi: https://doi.org/10.20944/preprints201905.0342.v1

15. Ramlau, R., Scherzer, O. (Eds.) (2019). The Radon Transform. Berlin/Boston: Walter de Gruyter GmbH. doi: https://doi.org/ $10.1515 / 9783110560855$

16. Li, Z., Liu, Y., Walker, R., Hayward, R., Zhang, J. (2009). Towards automatic power line detection for a UAV surveillance system using pulse coupled neural filter and an improved Hough transform. Machine Vision and Applications, 21 (5), $677-686$. doi: https://doi.org/10.1007/s00138-009-0206-y

17. Manzanera, A., Nguyen, T. P., Xu, X. (2016). Line and circle detection using dense one-to-one Hough transforms on greyscale images. EURASIP Journal on Image and Video Processing, 2016 (1). doi: https://doi.org/10.1186/s13640-016-0149-y

18. El-Baz, A., Jiang, X., Jasjit, S. (Eds.) (2016). Biomedical image segmentation: advances and trends. CRC Press, 546. doi: https://doi.org/10.4324/9781315372273

19. Ruban, I., Khudov, V., Makoveichuk, O., Khudov, H., Khizhnyak, I. (2018). A Swarm Method for Segmentation of Images Obtained from On-Board Optoelectronic Surveillance Systems. 2018 International Scientific-Practical Conference Problems of Infocommunications. Science and Technology (PIC S\&T). doi: https://doi.org/10.1109/infocommst.2018.8632045

20. Ruban, I., Khudov, H., Makoveichuk, O., Khizhnyak, I., Khudov, V., Podlipaiev, V. et. al. (2019). Segmentation of optical-electronic images from on-board systems of remote sensing of the earth by the artificial bee colony method. Eastern-European Journal of Enterprise Technologies, 2 (9 (98)), 37-45. doi: https://doi.org/10.15587/1729-4061.2019.161860

21. Ruban, I., Khudov, H., Khudov, V., Khizhnyak, I., Makoveichuk, O. (2017). Segmentation of the images obtained from onboard optoelectronic surveillance systems by the evolutionary method. Eastern-European Journal of Enterprise Technologies, 5 (9 (89)), $49-57$. doi: https://doi.org/10.15587/1729-4061.2017.109904

22. Dorigo, M., St tzle, T. (2018). Ant Colony Optimization: Overview and Recent Advances. International Series in Operations Research \& Management Science, 311-351. doi: https://doi.org/10.1007/978-3-319-91086-4_10

23. WorldView-1 Satellite Sensor. Satellite Imaging Corporation. Available at: http://www.satimagingcorp.com/satellite-sensors/ worldview-1

24. Ruban, I., Khudov, V., Khudov, H., Khizhnyak, I. (2017). An improved method for segmentation of a multiscale sequence of optoelectronic images. 2017 4th International Scientific-Practical Conference Problems of Infocommunications. Science and Technology (PIC S\&T). doi: https://doi.org/10.1109/infocommst.2017.8246367 